
and the area of highest seismic activity during the 2004-2008 seismic crises.

\section{Scattering images from autocorrelation functions of P-wave seismic velocity images: the case of Tenerife Island (Canary Islands, Spain)}

\author{
García-Yeguas, A. ${ }^{(1)(2)(7)}$, Sánchez-Alzola, A. ${ }^{(3)}$, De Siena, L. ${ }^{(4)}$, Prudencio, J. ${ }^{(5)(2) ~(7)}$, Díaz-Moreno, \\ A. ${ }^{(8)}$, Ibáñez, J. M. ${ }^{(6)(2)}$ \\ (1) Departamento de Física Aplicada, Universidad de Cádiz, Spain \\ (2) Instituto Andaluz de Geofísica. Universidad de Granada, Spain. \\ (3) Departamento de Estadística e Investigación Operativa, Universidad de Cádiz, Spain \\ (4) Departament of Geology and Petroleum Geology, University of Aberdeen, United Kingdom \\ (5) Earth and Planetary Science Department. University of California, Berkeley, USA \\ (6) Departamento de Física Teórica y del Cosmos. Universidad de Granada. Spain \\ (7) Instituto Volcanológico de Canarias (INVOLCAN). Tenerife, Spain \\ (8) Department of Earth, Ocean and Ecological Sciences. University of Liverpool. United Kingdom. \\ * Corresponding author. Tel.: +34956483320. e-mail address: araceli.garcia@uca.es
}

\section{Abstract}

We present a P-wave scattering image of the volcanic structures under Tenerife Island using the autocorrelation functions of $P$-wave vertical velocity fluctuations. We have applied a cluster analysis to total quality factor attenuation $\left(Q_{t}^{-1}\right)$ and scattering quality factor attenuation $\left(Q_{P S C}^{-1}\right)$ images to interpret the structures in terms of intrinsic and scattering attenuation variations on a $2 \mathrm{D}$ plane, corresponding to a depth of $2000 \mathrm{~m}$, and check the robustness of the scattering imaging. The results show that scattering patterns are similar to total attenuation patterns in the South of the island. There are two main areas where patterns differ: at Cañadas-Teide-Pico Viejo Complex high total attenuation and average-to-low scattering values are observed. We interpret the difference as induced by intrinsic attenuation. In the Santiago Ridge Zone (SRZ) region, high scattering values correspond to average total attenuation. In our interpretation, the anomaly is induced by an extended scatterer, geometrically related to the surficial traces of Garachico and El Chinyero historical eruptions 
34 Keywords: P-wave scattering images, Canary Islands, velocity and attenuation seismic tomography, autocorrelation functions.

36

\section{Introduction}

Since the second half of the Twentieth century different geophysical techniques have been developed to obtain direct images of the Earth's interior in a noninvasive way (e .g. seismic tomography (Aki et al. 1977); magnetotelluric (Cagniard 1953); gravimetry (Valliant 1991)). Among those employing travel times and amplitudes of seismic wave-packets, seismic tomography is the one that has evolved most over time, using different Earth properties as model parameters to represent the internal structure of the Earth and various types of observable signals as data (Aki et al. 1977; Nolet 2008).

The spatial scales resolved by using body-wave velocity tomography via measurements of Pand S-travel times can range from a few hundred meters in the crust (Patanè et al. 2017) to $500-1000 \mathrm{~km}$ in global tomography of the Earth mantle and core (Romanovicz, 2003; Nolet 2008). Seismic velocity tomography can be integrated by attenuation tomography, imaging the energy lost by P- and S-waves while traveling into the Earth (as quality factors Qp and Qs). With these techniques, researchers can better illuminate and interpret Earth features and characterize structural heterogeneities (Schurr et al. 2003; De Gori et al. 2005; EberhartPhillips et al. 2008). Attenuation is, in fact, more sensitive than velocity to strong spatial changes in the Earth composition, temperature, and pressure (Romanowicz, 2003) and an excellent marker of time-dependent changes in the physical and chemical properties of the medium (O'Connell and Budiansky, 1977; Fehler, 1982; Gusev and Lemzikov, 1985; Fehler et al., 1988).

At volcanic scale, integrated P- and S-wave velocity (Vp and Vs) and attenuation tomography models have therefore been increasingly successful at imaging volcanic structures (De Gori et al. 2005; De Siena et al. 2014; Prudencio et al. 2015a, 2015b). Still, we do not fully 
understand how seismic waves behave when interacting with extreme structural heterogeneity, thus greatly increasing the difficulty of modeling velocity and attenuation with deterministic physical theories and direct wave information (Sato et al.2012). Seismic coda waves (the late portion of an earthquake recording) have thus acquired increasing importance in active volcanoes to detect small-scale heterogeneity and magma plumbing systems (Sato et al. 2012; De Siena et al. 2014; De Siena et al. 2016). By using these secondary incoherent arrivals in the seismogram, total attenuation may be separated into intrinsic attenuation (energy dissipated by the seismic waves while travelling through the medium) and scattering attenuation (energy lost due to the interaction of the seismic waves with the medium heterogeneities, and effectively recorded later in the seismogram as coda). Several methods have been devised to separate and image these two contributions to total attenuation (Hoshiba et al., 2001; Akinci et al., 1995; Del Pezzo et al., 2001, 2006; Giampiccolo et al., 2006). In one of the first applications of scattering attenuation imaging, Nishigami (1997) found strong scattering bodies beneath volcanic areas, extending to $7 \mathrm{~km}$ (Mount Ontake volcano, Japan) and $20 \mathrm{~km}$ depths (Mount Nikko-Shirane volcano, Japan), respectively. The presence of a vertical region of high scattering under both volcanoes was interpreted as a magma conduit. Mikada et al (1997) found several patches of strong scatterers beneath Izu-Oshima volcano (Japan), which were interpreted as the primary magma reservoir. Revenaugh (1997) provides a review of the first scattering imaging studies applied to imaging geologic media. In the early 2000s, different techniques have been developed to obtain 2D and 3D intrinsic and scattering attenuation models for S-waves in volcanic areas (Frederiksen and Revenaugh 2004; Tramelli et al. 2006; Carcolé and Sato 2010; De Siena et al. 2014; De Siena et al. 2016). In particular, Prudencio et al. (2013) have used a diffusive approximation (Wegler and Lürh, 2001) to obtain intrinsic and scattering maps of Tenerife Island. To apply any of the

84 above-mentioned methods it is necessary to use seismic waveforms. 
When a $\mathrm{P}$ and $\mathrm{S}$ traveltime dataset is available but waveforms are too noisy to provide direct attenuation measurements, scattering attenuation measurements can be obtained using P-and $\mathrm{S}$-wave velocity models. This method infers the scattering properties of $\mathrm{P}$ - and/or $\mathrm{S}$-waves from three-dimensional velocity fluctuations, obtained from a velocity model (De Siena et al. 2011; Sato et al. 2012). The technique is based on the fact that, at scales smaller than a few hundred meters, velocity fluctuations tend to be random (Holliger et al. 1996). They can thus be obtained by using the spatial autocorrelation functions (ACF) of vertical velocity fluctuations calculated at different lateral points of a 3D velocity model (De Siena et al. 2011).

Among all possible statistical distributions, Von Karman and exponential distributions fit properly the velocity fluctuations. In fact, for the shape parameter $\lambda=0.5$ both distributions coincide. However, for exponential distribution the correlation length is directly related to the dominant characteristic scales lengths of the heterogeneities in the medium (Yoon, 2005). We need the correlation length (a) to make our interpretation and obtain scattering quality factor attenuation $\left(Q_{P S C}^{-1}\right)$. It is today accepted the exponential distribution formodeling velocity fluctuations in either a stratified or volcanic medium, providing a statistical measure of the spatial scale and magnitude that characterizes heterogeneities in a volcano (Holliger et al. 1996; Shapiro and Hubral 1999, De Siena et al. 2013). In the Jemez volcanic field, spatial exponential ACFs of 3D velocity fluctuations are able to model strong stratification, image velocity changes when they are stronger in the vertical with respect to the horizontal direction, and identify a scattering anomaly beneath the volcanic cone (Sato et al. 2012). If used jointly with direct total attenuation measurements and interpreted via Cluster Analysis (CA), as at Campi Flegrei caldera (Italy) and Mount St. Helens volcano (USA), ACFs can be used to image and interpret scattering and intrinsic attenuation in 2D (De Siena et al. 2011, 2016). 
110 Here, we have applied this technique to the island of Tenerife (Canary Islands, Spain), where

111 models of velocity (García-Yeguas et al. 2012), total quality factor attenuation $\left(Q_{t}^{-1}\right)$

112 (Prudencio et al. 2015a), and scattering and intrinsic attenuation of S-waves (Prudencio et al.

113 2013) are available. The aim of this work is to map $Q_{P S C}^{-1}$ at Tenerife Island in a 2D plane at

$114 \sim 2000 \mathrm{~m}$ of depth and apply a CA to this and total attenuation measurements. We thus: (1)

115 provide a new P-wave scattering image of the Island and (2) check the robustness of the

116 method and interpret the results by comparing them with different seismic models. We have

117 first calculated the ACFs of vertical velocity fluctuations measured from the P-wave seismic

118 velocity model of Tenerife Island (Garcia-Yeguas 2010; Garcia-Yeguas et al. 2012) between

119 depths of $100 \mathrm{~m}$ and $4500 \mathrm{~m}$. The parameters controlling the ACFs are the correlation length

120 (a) and the mean square fractional fluctuations $\left(\varepsilon^{2}\right)$ : these are used to calculate and map $Q_{P S C}^{-1}$

121 at a depth of $\sim 2000 \mathrm{~m}$ assuming a travel time-corrected Born approximation (Aki and

122 Richards 1980; Sato and Fehler 2012). Finally, we applied a CA to the images of $Q_{P S C}^{-1}$

123 (obtained in this work) and $Q_{t}^{-1}$ obtained by Prudencio et al. (2015a) at the corresponding

124 depth, to interpret the scattering image and check the robustness of the results. The

125 comparison of the results with the volcanic eruptive history, the known geological structures,

126 and the recent seismic activity unravels important variations in the lateral structure and

127 dynamics of the volcanic area.

128

1292 Geological settings

130 Tenerife Island is a volcanic island that belongs to the Canary Islands archipelago (Spain)

131 (Figure 1). It is the major island of the Canary Islands with an area of $2,040 \mathrm{~km}^{2}$. Its

132 geomorphology is very heterogeneous, with monogenetic cones, basaltic and felsic lava

133 flows, stratovolcanoes, domes, and pyroclastic deposits; evidence of diverse volcanic 
135 The most significant volcanic complex is located in the center of the island and comprises 136 Las Cañadas caldera, an elliptical $16 \times 9 \mathrm{~km}$ caldera lying at $2000 \mathrm{~m}$ above sea level (LCC in

137 figure 1), within which lies a stratovolcanic complex identified as Teide-Pico Viejo (Teide138 PV in figure 1). Together these structures form the Cañadas-Teide-Pico Viejo Complex 139 (CTPVC in figure 1).

140 Different periods of activity have been identified in the evolution of CTPVC evolution, 141 separated by longer intervals of quiescence (Araña et al. 1994). CTPVC has two rift zones: 142 the Santiago Rift Zone (SRZ in Figure 1) to the NW and the Dorsal Rift Zone (DRZ in Figure 143 1) to the NE. The last historical eruptions took place, with the exception of the Chahorra 144 eruption, that occurred in 1798 within the CTPVC complex, all historical eruptions have 145 taken place along these two rift zones (Romero 1991, 1992). Sietefuentes (1704), and Fasnia 146 and Arafo (1705), and the SRZ eruptions have been Garachico volcano (1706) and El 147 Chinyero (1909).

148 Several studies have imaged the inner structure of Tenerife Island using different physical 149 properties: resistivity measurements (Pous et al. 2002; Coppo et al. 2008; Piña-Varas et al. 150 2014; Piña-Varas et al. 2015); aeromagnetic surveying (Blanco-Montenegro et al. 2011), 151 gravity (Araña et al. 2000; Gottsmann et al. 2008) and seismological studies (Canales et al. 152 2000; García-Yeguas 2010; García-Yeguas et al. 2012; Prudencio et al. 2013, 2015a). 




154 Fig. 1: Regional setting and location of Tenerife Island. Rift position and Las Cañadas wall 155 are marked with yellow lines. CTPVC: Cañadas-Teide-Pico Viejo Complex, SVZ: Southern 156 Volcanic Zone, LCC: Las Cañadas Complex, PV: Pico Viejo, MB: Montaña Blanca, BTV: 157 Boca Tauce Volcano, SRZ: Santiago Rift Zone, RdC: Roque del Conde, DRZ: Dorsal Rift 158 Zone and FASF: Fasnia-Arafo-Siete Fuentes eruption.

3 Data and Methodology

162 To perform this study we have used the high-resolution 3D P-wave seismic velocity and 163 attenuation models of Garcia-Yeguas et al. (2012) and Prudencio et al. (2015a). Both models were obtained using the seismic recordings of an active seismic experiment (TOM-TEIDEVS - Ibáñez et al. 2008). More than 6300 shots were fired by air guns connected to a BIO 166 Hespérides research vessel and recorded at 125 seismometers deployed onland. A subset of 167103,750 high-quality travel times and waveforms were chosen to perform the tomographic inversions. The velocity model has lateral extension of $40 \times 40 \mathrm{~km}^{2}$ and depth ranging from 
the top of Teide volcano (over $3000 \mathrm{~m}$ above sea level (a.s.l.)) to $8000 \mathrm{~m}$ below sea level (b.s.1.) at a node spacing of $0.7 \mathrm{~km}$ in all directions.

171 To obtain the ACF of vertical velocity fluctuations and derive a $Q_{P S C}^{-1}$ image at a depth of $172 \sim 2000 \mathrm{~m}$ we have selected the regions of maximum resolution and reliability between depths 173 of $0.1 \mathrm{~km}$ and $4.5 \mathrm{~km}$, following the resolution and reliability tests of the two tomographic studies (see Garcia-Yeguas et al. (2012) and Prudencio et al. (2015a) for details). We have applied a CA using as input parameters the values of $Q_{P S C}^{-1}$ and the values of $Q_{t}^{-1}$ obtained by Prudencio et al. (2015a) at a depth of $2000 \mathrm{~m}$.

177 The magnitude and scale of random heterogeneities in a volcanic medium can be quantified by the ACF of velocity fluctuation distributions (Aki and Chouet 1975). The $a$ and the $\varepsilon^{2}$ control the shape and amplitude of the ACF and are sensitive to the physical state of the upper crust (Sato and Fehler 2012). Here, we have followed the methodology described by De Siena et al. (2011), using as data the 3D velocity measurements obtained by GarcíaYeguas et al. (2012). These data are distributed on a regular grid of $0.7 \times 0.7 \times 0.7 \mathrm{~km}$ step, providing seven vertical high-resolution velocity measurements at each point on the $2 \mathrm{D}$ map.

We fit a $\mathrm{N}^{\text {th }}$-order average polynomial over the seven vertical measurements at each point on the map to remove the vertical trend and randomize the velocity values. We determine the polynomial degree of the generic velocity function as:

$$
V(z)=a_{0}+a_{1} z+a_{2} z^{2}+\ldots+a_{N} z^{N}
$$

This is obtained using the Bayesian (or Schwartz, 1978) Information Criterion (BIC) and considering equal variance values $\widehat{\sigma_{e}^{2}}$ at all nodes:

$$
\mathrm{BIC}=n \ln \left(\widehat{\sigma_{e}^{2}}\right)+\mathrm{N} \ln (n)
$$

191 where $\widehat{\sigma_{e}^{2}}$ is the error variance for normally distributed errors, $\mathrm{N}$ is the number of model parameters to be obtained (polynomial degree) and $n$ the number of observations. A higher 
194 introducing a penalty term against the increasing number of parameters. After averaging the 195 BIC results over the entire resolved map, and using the elbow method (Hartigan 1975), we 196 observe that a first order polynomial (or a simple regression) with two parameters:

$$
V(z)=a_{0}+a_{1} z
$$

198 This provies a best fit the vertical trend at best. Figure 2 a shows the BIC as a function of the polynomial degree. We observe that polynomial degrees greater than one do not improve significantly the BIC values.
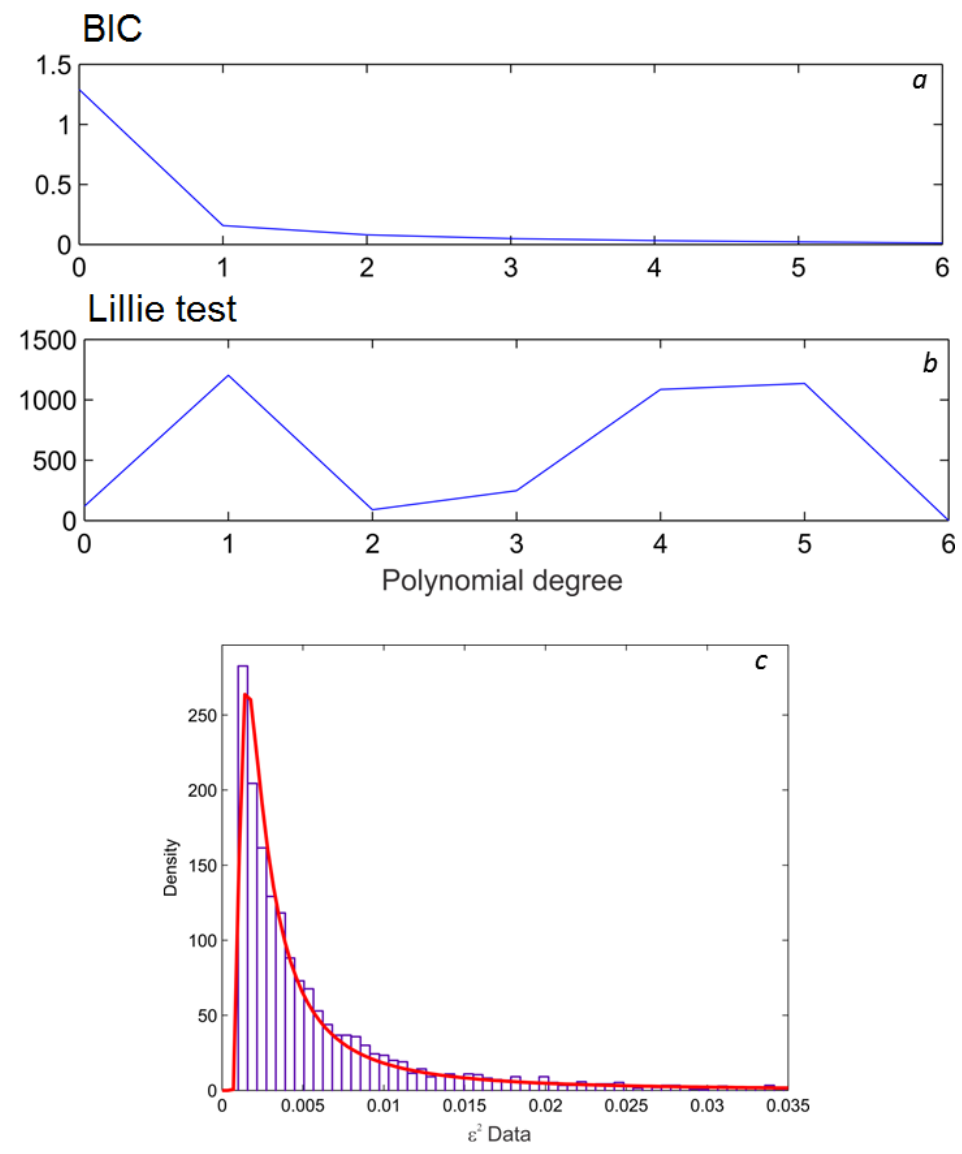

203 Fig. 2: a. The BIC criterion as a function of polynomial order. b. Number of de-trended 204 random Gaussian measurements on the map showing random fluctuations after de-trending 205 and a Lilliefors test (Lilliefors, 1967) c. Histogram of the $2 \mathrm{D} \varepsilon^{2}$ spatial variations fitted with a 206 Generalized Extreme Value distribution (GEV - red line). 
208 In a perfectly random medium the velocity fluctuations should be random after de-trending.

209 We have applied the Lillie test (Lilliefors, 1967) at 5\% confidence to the velocity fluctuations

210 at all points on the map after de-trending. The null hypothesis is that the data distribution is

211 normal. For different polynomial degrees, we store the points having a p-value (probabilities

212 that support the null hypothesis) greater than the significance level of 0.05 , where the null

213 hypothesis is not rejected. In figure $2 \mathrm{~b}$ we plot the number of points that passed the test

214 against the order of the polynomial used for de-trending. The highest number of points

215 presenting random Gaussian fluctuations corresponds to the lowest degree in the polynomial

216 (first order polynomial). Our aim is to obtain the maximum number of random points using

217 the polynomial with the lowest polynomial degree $\mathrm{N}$. The values of Lillie test for $\mathrm{N}=1$ and

218 N=4-5 shown in Fig 2 are fairly similar, with maximum number of blocks around 1200-1300;

219 however, only polynomials of $\mathrm{N}=1$ and $\mathrm{N}=2$ provide comparable values for the $\mathrm{BIC}$. If we had obtained a similar amount of random blocks with $\mathrm{N}=1$ and $\mathrm{N}=2$ with the Lillie test, our choice of a polynomial of order 1 would have been unjustified. As there is an increase in number of random blocks only for order $\mathrm{N}=4$, the best choice is $\mathrm{N}=1$. The de-trend of the velocity model in the area is thus done using best-fit coefficients for the polynomial:

$$
\tilde{v}^{*}(z)=V^{*}(z)-a_{0}^{*}-a_{1}^{*}(z)
$$

where $\tilde{v}^{*}(z)$ are the velocity fluctuation distribution with depth for any surface grid point $(\mathrm{x} *$, $\mathrm{y} *)$. The normalized $\mathrm{ACF}, \mathrm{R}(\Delta \mathrm{z})$, is calculated numerically as:

$$
R(z)=\left\langle\tilde{v}^{*}(z) \tilde{v}^{*}(z+\Delta z)\right\rangle
$$

where the brackets indicate spatial average and $\Delta \mathrm{z}$ is the correlation space-lag positive for the increasing depth. Additionally, we have used an exponential ACF model to adjust the random velocity fluctuation (Shapiro and Hubral, 1999). We show some examples of ACF values and its fit in the Supplementary Material (figure S1). This model depends on $\varepsilon^{2}$ and $a$ as: 


$$
R(z)=\varepsilon^{2} \exp (-z / a)
$$

233 where the maximum of $R(z)$ coincides with $\varepsilon^{2}$ at $z=0$. We compute the values of space 234 averaged $a$ as being $1.968 \pm 0.013 \mathrm{~km}$ and the space-averaged value $\varepsilon^{2}$ is $0.00365 \pm 0.00015$ 235 (these values have been computed with a confidence of 95\%). We also fit a Generalized 236 Extreme Value distribution (Fréchét distribution) to the $\varepsilon^{2}$ values obtained at all grid points on the map. This distribution has been shown to fit the velocity fluctuations as a sequence of non-gaussian independent and identically distributed random variables and in the presence of strong heterogeneity (De Siena et al. 2011; De Siena et al. 2016). Figure 2c shows the fit of the $2 \mathrm{D} \varepsilon^{2}$ with a generalized extreme value distribution with a shape parameter $\lambda$ of 0.000815. As De Siena et al. (2011), we remark that the $a$ and the $\varepsilon^{2}$ are biased. We also note that the $a$ measurements are affected by larger uncertainties.

243 Both $\varepsilon^{2}$ and a parameters are used to model $\mathrm{Q}^{-1}$ scattering for P-waves $\left(Q_{P S C}{ }^{-1}\right)$. We have 244 followed the theory of elastic random fluctuations in the Born approximation assuming an exponential ACF. This approximation considers a cut-off wavenumber value $\left(v_{c}\right) v_{c}{ }^{2}=1 / 4$ to correct high-frequency dependencies at a determined wave number $(\kappa)$ (De Siena et al., 2011;

247 Sato and Fehler, 2012).

$$
Q_{P S C}^{-1}=\frac{15 \varepsilon^{2} a^{3} \kappa^{3}}{2\left(1+\frac{a^{2} \kappa^{2}}{4}\right)\left(1+4 a^{2} \kappa^{2}\right)}
$$

where $\kappa=2 \pi f / v, \mathrm{f}$ is frequency in $\mathrm{Hz}$ and $\mathrm{v}$ is the $\mathrm{P}$-wave seismic velocity in $\mathrm{km} / \mathrm{s}$. In this study we have chosen $\mathrm{f}=6 \mathrm{~Hz}$ as this is the central frequency used to filter the seismic signals to perform the velocity tomography (García-Yeguas et al. 2012), and we have assumed $v=6 \mathrm{~km} / \mathrm{s}$ as the average P-wave seismic velocity of the model (García-Yeguas et al. 2012). Finally, we have applied a K-means cluster analysis method to relate in space the variations of the $Q_{P S C}{ }^{-1}$ and with those of the total quality factors $\left(Q_{P t}{ }^{-1}\right)$ (Hartigan 1975; 
256 low $Q_{P S C}{ }^{-1}$ and $Q_{P t}^{-1}$ (two options for each variable), picking four clusters makes

257 interpretation of the anomalies easier. This is only true if the difference in reduction in point-

258 to-centroid distance of four clusters with respect to three or five is small, as in our case.

259 Previously we have made a statistical analysis comparing both variables to check unreliable

260 spatial measurements of $Q_{P S C}{ }^{-1}$ and $Q_{P t}{ }^{-1}$ dispersion. This study is included in the

261 Supplementary Material (figure S2). We have used the Euclidean distance to group the two

262 different variables in their parameter space: $Q_{P S C}{ }^{-1}$ obtained in this study, and $Q_{P t}{ }^{-1}$

263 measured by Prudencio et al. (2015a) at $2000 \mathrm{~m}$ b.s.l. To choose the number of clusters, we

264 plot the percentage reduction in point-to-centroid distance against the number of clusters

265 (Figure 3a). The ideal number of clusters is that after which the distance is not consistently

266 improved increasing number of clusters i. e. the point after which the curve starts a plateau

267 and/or goes over a set threshold (80\%). The elbow method suggests the need of $\mathrm{K}=4$

268 clusters, at the start of the plateau of percentage reduction and where we achieve almost $80 \%$

269 reduction of the cluster-to-observation distance (De Siena et al. 2011; De Siena et al. 2016).

270 Figure $3 \mathrm{~b}$ shows the variables (dots) in their parameter space, labeled with a color

271 corresponding to each cluster. A crossed circle marks the cluster centroids. We observe that

272 red plots are in regions where scattering attenuation is larger than total attenuation. This is

273 due to the use of two different methodologies to obtain total attenuation - Prudencio et

274 al.(2015) and scattering attenuation (De Siena et al., 2011). The inverse total quality factor

275 cannot be retrieved by the sum of the scattering and intrinsic quality factor exactly with this

276 analysis, as stated in De Siena et al. (2011). The results can only be interpreted qualitatively

277 using clusters analysis. Furthermore, the K-means algorithm also classifies the data by taking

278 into account the point-to-centroid distance only, without taking into account other aspects

279 (such as geological formations and the inner structure of the volcano) which could better

280 constrain our analysis. 




282

283

284

285

286

287

288

289

290

291

292

293

294

295

296

297

\section{Results and discussion}

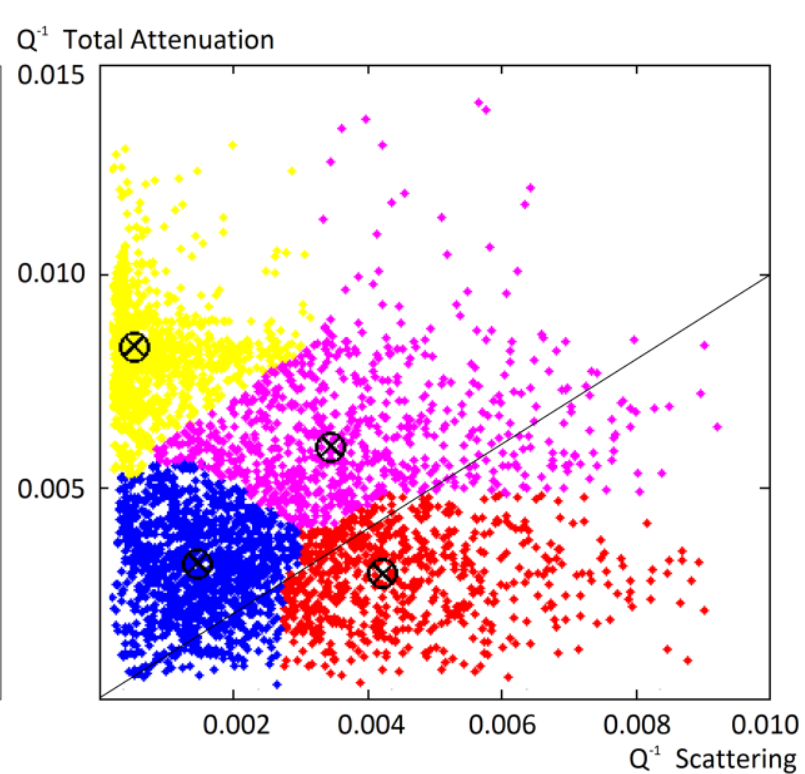

Fig. 3: a. Percent reduction in point-to-centroid distance with respect to the number of clusters. The black line shows the $80 \%$ reduction obtained with 4 clusters. b. Results of the $4-$ means CA . A black-crossed circumference marks the centroids. The black line divides the image in two sections. The points located in the lower section are considered erroneous because $Q_{P S C}{ }^{-1}$ is greater than $Q_{P t}{ }^{-1}$. The percentage of erroneous points is $10.94 \%$ in the studied area (from the original grid $40 \mathrm{~km} \times 40 \mathrm{~km}$ ). The most affected cluster is the red one.

We assume these regions are affected by anomalously high scattering.

Based on the checkerboard tests carried out by García-Yeguas et al. (2012), we have chosen only the region of the island above sea level, where the resolution is higher in this area. To provide a first qualitative interpretation of the scattering image, and check the robustness of the method, we compare the $\varepsilon^{2}$ and $Q_{P S C}^{-1}$ images with the P-wave total attenuation model (Prudencio et al. 2015a). The $a$ parameter values vary from $\sim 0.37 \mathrm{~km}$ to a maximum of 2.91 $\mathrm{km}$. However, De Siena et al. (2016) pointed out that this variable is influenced by huge 
uncertainties; therefore, we consider $\varepsilon^{2}$ as the best parameter to model P-waves heterogeneities at Tenerife Island.

300

3014.1 Spatial patterns of $\varepsilon^{2}$ and $Q_{P S C}^{-1}$.

302 In figure 4 we show the $2 \mathrm{D}$ measurements of $\varepsilon^{2}$ and derived $Q_{P S C}^{-1}$ super-imposed on a

303 contour-map of Tenerife Island. The spatial patterns of $\varepsilon^{2}$ identify areas of high scattering

304 heterogeneity and control seismo-volcanic coda envelope shapes at intermediate and late

305 lapse times (De Siena et al. 2013). Figure 4a shows high $\varepsilon^{2}$ values in the Santiago Rift Zone

306 (SRZ) and medium and low values across the rest of the island.

307 The highly-heterogeneous $\varepsilon^{2}$ patterns in the SRZ are spatially correlated to historical 308 eruptions in this area (Romero 1991, 1992) including Garachico (occurred in 1706) and El 309 Chinyero (in 1909, the last eruption occurred in Tenerife). The $\varepsilon^{2}$ values, related to the mean 310 squared fractional fluctuation, are estimated in this study as varying from 0.00 to 0.12 . These 311 values are also consistent with other volcanic studies such as De Siena et al. (2011). We 312 consider that high values of $\varepsilon^{2}$ could be related to either the presence of non-consolidated and 313 fragmented materials or residual hot magmatic intrusions, as they generally correspond to 314 low P-wave velocities (García-Yeguas et al. 2012) and density values (Gottsmann et al. 315 2008). The seismicity recorded between 2004 and 2008 is the expression of a general 316 volcanic reactivation and mainly affects NW sector of the island (Almendros et al. 2007;

317 Cerdeña et al. 2011). While high $\varepsilon^{2}$ in this region corresponds to the presence of cinder cones, 318 historical eruptions, and recent seismicity (figure 4a), the relationship of high $\varepsilon^{2}$ to geological 
319 heterogeneities and seismic activity is unclear in the rest of the island.
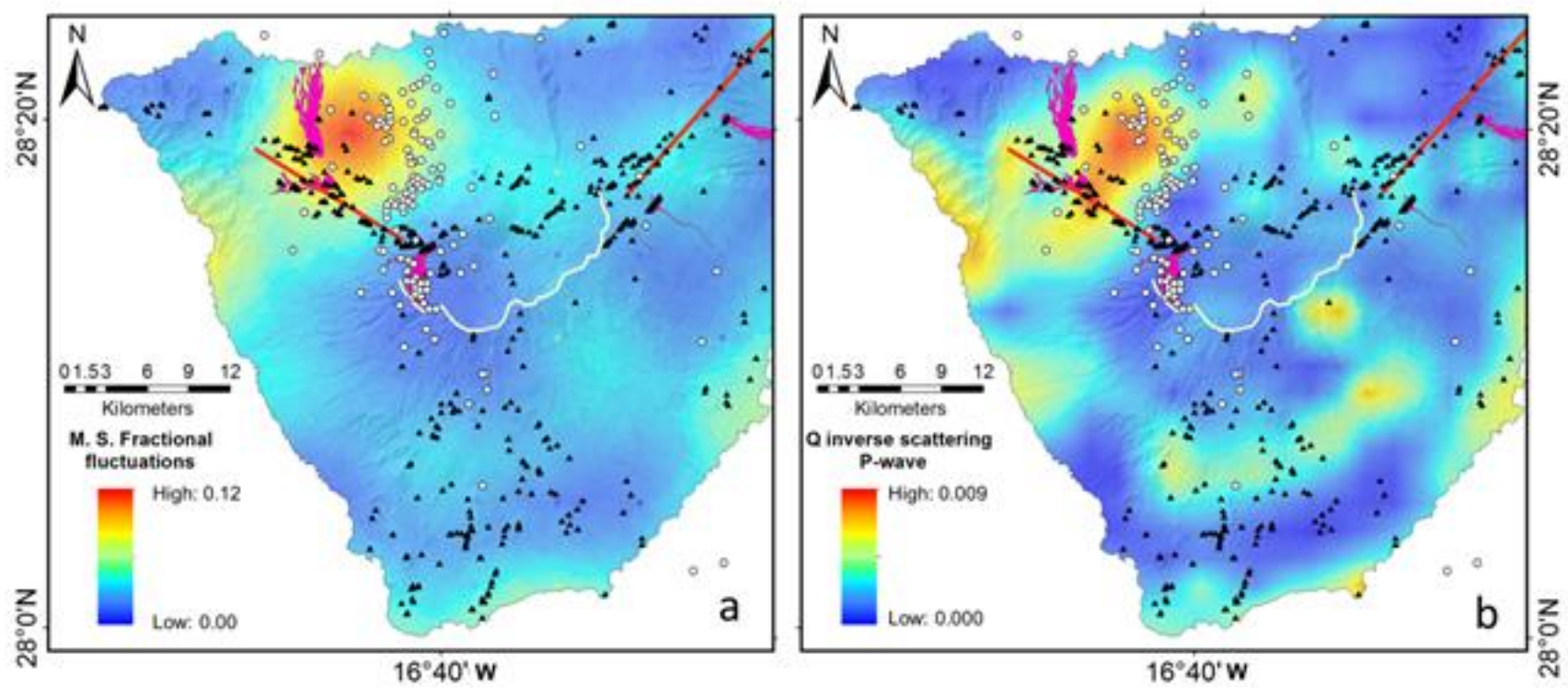

321 Fig. 4: a. Spatial patterns of $\varepsilon^{2}$ measured from the ACF distribution. b. P-wave $\mathrm{Q}^{-1}$ scattering.

322 White circles indicate the epicenters of the earthquakes between 2004 and 2008 (from 323 Cerdeña et al. 2011). Black triangles and purple areas mark cinder cones and historical 324 eruptions, respectively. Red lines indicate SRZ (left) and DRZ (right). The white line outlines the rim of Las Cañadas Caldera.

327 The map of inverse quality factor for P-waves $\left(\mathrm{Q}_{\mathrm{PSc}}^{-1}\right.$, figure $\left.4 \mathrm{~b}\right)$ highlights regions showing different scattering characteristics: i) the SRZ, southeastern and southern edges of the island are characterized by the highest values of $Q_{P S C}^{-1}$, ii) DRZ and southeastern parts of the island display average $Q_{P S C}^{-1}$ values, and iii) the central, south-central, and north-easternmost parts of

331 the island show low $Q_{P S C}^{-1}$ values. High values of $Q_{P S C}^{-1}$ at the SRZ are correlated with low P-

332 wave velocity and density values at depth (Gottsmann et al. 2008; García-Yeguas et al. 333 2012). While these results hint atfluid and melt accumulation at depth, the region is also characterized at the surface by unconsolidated products of the last historical eruptions in 
335 Tenerife (Garachico in 1706 and El Chinyero in 1909), which may affect coda recordings at 336 frequencies characteristic of local earthquake tomography (De Siena et al. 2016). Prudencio

337 et al. (2013) obtained $Q^{-1} \mathrm{~S}$-wave scattering attenuation maps from the inversion of the 338 energy envelopes recorded at different source-receiver pairs, showing a similar--although 339 damped--high-scattering anomaly in the area. East of the anomaly, the seismicity that 340 occurred between 2004 and 2008 matches with high $Q_{P S C}^{-1}$ values in this region. The 341 hypocenters of the earthquakes in Northwest Tenerife are mainly located between depths of 0 342 and $6 \mathrm{~km}$ (Cerdeña et al. 2011) and our map is produced for a depth of $2000 \mathrm{~m}$ using data 343 from $0.1 \mathrm{~km}$ to $4.5 \mathrm{~km}$ depths. We relate the seismicity with an unconsolidated area in the 344 North of the island, and high values at the southeastern and southern edges of the island could 345 be related to fragmented or/and unconsolidated material at these locations.

347 The DRZ and Southeast of Tenerife display medium-high values of $Q_{P S C}^{-1}$. We associate the 348 anomalies in the DRZ with unconsolidated material of the last eruptions in 1704 (Siete 349 Fuentes) and 1705 (Fasnia). In the southeastern part of the island there has been no recent 350 volcanic activity, thus we associate this $Q_{P S C}^{-1}$ values with volcanoclastic deposits and 351 fragmented material. On the other hand, the central, south-central, and north-easternmost parts of the island have low $Q_{P S C}^{-1}$ values. We relate these areas to consolidated cool nonfragmented bodies. This interpretation is in agreement with the presence of a high-velocity (García-Yeguas et al. 2012) and high-density (Gottsman et al. 2008) body in the center of Tenerife.

358 Figure 5c shows the results obtained from the CA using the values of $Q_{P S C}^{-1}$ obtained in this study (figure 5a) and the total attenuation tomography model provided by Prudencio et al. 
360 (2015a) (figure 5b). The result provides a quantitative way to interpret attenuation in terms of 361 intrinsic and scattering mechanisms on a 2D plane corresponding to a depth of $\sim 2000 \mathrm{~m}$. The 362 CA patterns show (figure 5c):

363 - Regions in pink (high $Q_{P S C}^{-1}$ and high $Q_{t}^{-1}$ ): these regions are characterized by high 364 values of both $Q_{P S C}^{-1}$ and $Q_{t}^{-1}$. In Figure 5c, the pink areas located outside of Las 365 Cañadas were identified as high intrinsic attenuation regions by Prudencio et al. 366 (2013). Following Prudencio et al. (2013), we interpret these anomalies has being induced by fragmented materials and/or volcanoclastic deposits.

- Regions in blue (low $Q_{P S C}^{-1}$ and low $Q_{t}^{-1}$ ): Neither scattering nor intrinsic attenuation dominate these areas. We infer these regions are mainly comprised of well consolidated materials (Del Pezzo, 2008; Prudencio et al. 2017).

- Regions in yellow (low $Q_{P S C}^{-1}$ and high $Q_{t}^{-1}$ ): In these regions, P-wave attenuation is mainly due to intrinsic mechanisms. Some authors (Mayeda et al. 2008; Prudencio et al. 2013, for example) highlight that attenuation in volcanic regions is generally produced by scattering processes. The intrinsic absorption dominates over the scattering attenuation for regions where low seismic activity occurs. We observe that these regions are located in the outer part of the island and there is not seismic activity in them (Canas et al. 1998).

- $\quad$ Regions in red (high $Q_{P S C}^{-1}$ and low $Q_{t}^{-1}$ ): Northeast of CTPVC, the SRZ and DRZ are all characterized by anomalously high scattering attenuation. In figure $3 b$ we can observe that most of the attenuation values in this cluster are mainly due to scattering, in comparison with the pink cluster. De Barros et al. (2012) identified these regions as being associated with ancient magmatic bodies which fed eruptions of the last 2000 years in CTPVC. In addition, high values of scattering and measurable E-W 
compression (Sánchez-Alzola et al. 2016) can be related to the presence of fragmented materials mixed with consolidated bodies. They maybe related to dikes and ancient conduits of the last historic volcanic eruptions (Boca Cangrejo, 1492; Garachico, 1706; Chinyero, 1909). In the South of Tenerife Island there are also small patterns of high $Q_{P S C}^{-1}$ and low $Q_{t}^{-1}$. As there is no evidence of recent volcanic activity, these anomalies are more feasibly related to old volcanoclastic deposits combined with ancient consolidated constructs.
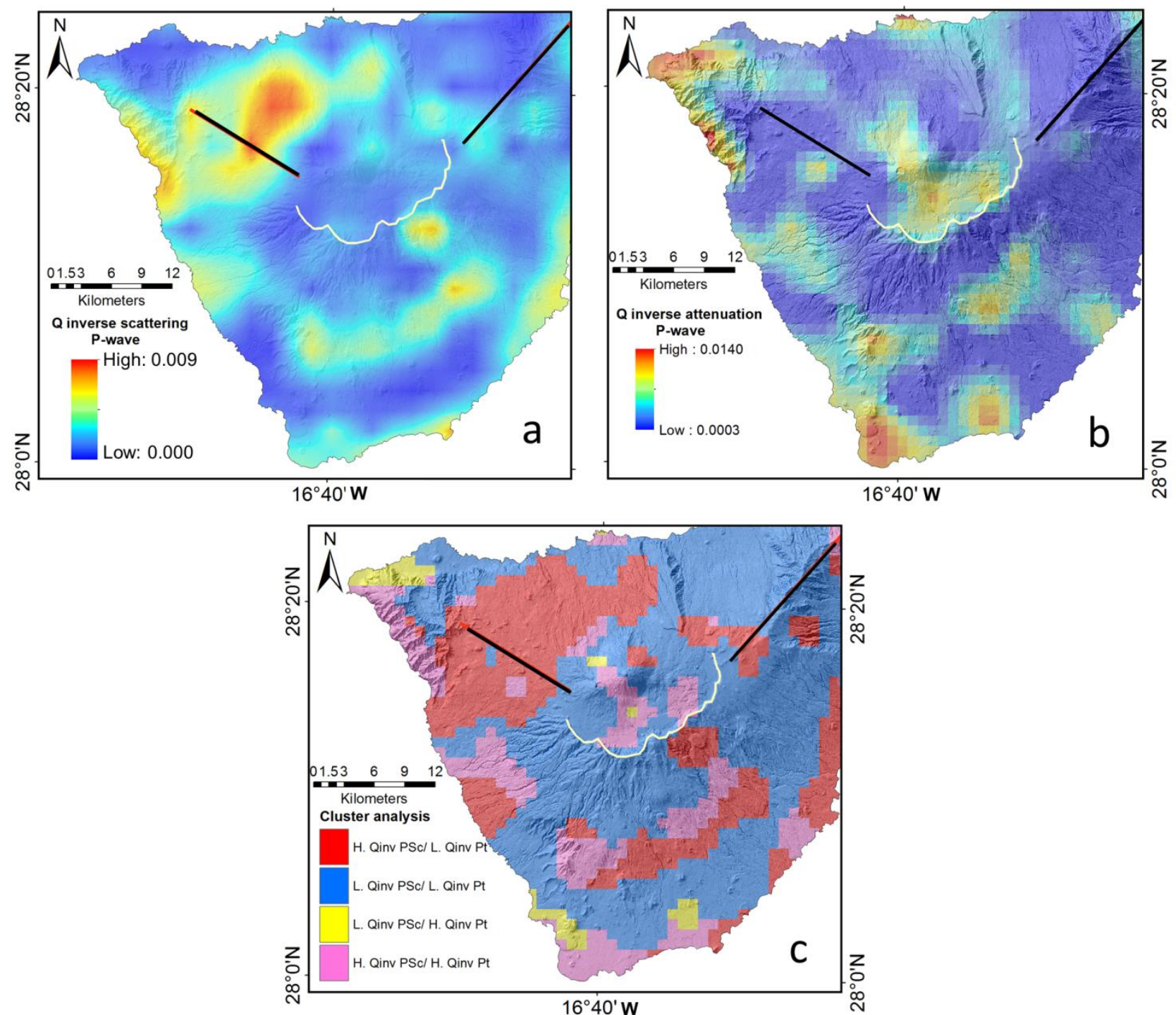
392 Fig. 5:a. $Q_{P S C}^{-1}$ map, b. $Q_{t}^{-1}$ map, and c. CA distribution. Red: High $Q_{P S C}^{-1}$ and low $Q_{t}^{-1}$. Blue:

393 Low $Q_{P S C}^{-1}$ and low $Q_{t}^{-1}$. Yellow: Low $Q_{P S C}^{-1}$ and high $Q_{t}^{-1}$. Pink: High $Q_{P S C}^{-1}$ and high $Q_{t}^{-1}$. 394 Black lines indicate SRZ (left) and DRZ (right). White line designates Las Cañadas Caldera 395 wall.

\section{Conclusions}

In this work, a P-wave scattering $\left(Q_{P S C}^{-1}\right)$ map has been obtained from the vertical autocorrelation functions (ACF) of measured spatial velocity variations. The $Q_{P S C}^{-1}$ distribution is obtained by using the measured $a$ and $\varepsilon^{2}$ in a single scattering approximation.

The method is particularly valid when seismograms are either unavailable or difficult to read.

402 The results confirm that the acquisition of a scattering image from a tomographically defined 403 velocity model increases our ability to interpret active volcanic structures. The CA technique 404 is applied to scattering and total attenuation anomalies to separate scattering and intrinsic attenuation on a 2D map at a depth of $\sim 2000$ m quantitatively; the comparison with literature and volcanological information shows the robustness of the technique, particularly in

407 retrieving high-scattering, magma-related anomalies.

408 Focusing on $\varepsilon^{2}$ and a, we note that high $\varepsilon^{2}$ values can be associated with high correlation 409 length values (De Siena et al. 2011). The scattering distribution is similar to the total 410 attenuation distribution in the South of the island. In other parts of Tenerife, we observe two 411 alternative settings. First at CTPVC low/average scattering attenuation corresponds to high 412 total attenuation. We infer from these values that the caldera is filled by unconsolidated rocks 413 and fragmented and fractured material from Teide-Pico Viejo stratovolcanoes (Coppo et al. 414 2008; Coppo et al. 2010; Piña-Varas et al. 2014; Prudencio et al. (2015a); and Villasante415 Marcos et al. 2014). Second at SRZ the high scattering attenuation is paired with low/average 
416 total attenuation. We relate these characteristics to the existence of a strong extended scatter,

417 linked with past volcanic (historical eruptions: Garachico and El Chinyero) and seismic 418 activity (seismic crisis of 2004-2008).

419

420 Acknowledgements

421 We thank Edoardo Del Pezzo for the valuable idea of this paper and suggestions regarding 422 the methodology. J. Prudencio is partially supported by NSF1521855 Hazard SEES project. 423 This paper has been partially supported by the Spanish project KNOWAVES (TEC2015424 68752-R (MINECO/FEDER)), the European project MED-SUV funded by the European 425 Union's Seventh Framework Program for research, technological development and 426 demonstration under Grant Agreement No 308665, and by the Regional project 'Grupo de 427 Investigación en Geofísica y Sismología de la Junta de Andalucía, RNM104’.

428

429

References

430

Ablay G J, Ernst G G J, Martí, J, Sparks R S J (1995) The 2 kasubplinian eruption of 431 Montaña Blanca, Tenerife. Bull Volcanol 57: 337-355.doi:10.1007/BF00301292.

432

433

434

435

436

437 438

Aki K, Christofferson A, Husebye E S (1977) Determination of the three-dimensional seismic structure of the lithosphere. $J$ Geophys. Res., 82, 277-296. Doi: 10.1029/JB082i002p00277

Aki K, Chouet B(1975). Origin of coda waves: source, attenuation, and scattering effects, $J$. Geophys. Res., 80, 3322-3342.

Aki K, Richards P (1980) Quantitative Seismology - Theory and Methods.W.H. Freeman, San Francisco. 
439 Akinci A, Del Pezzo E, Ibañez J (1995). Separation of scattering and intrinsic attenuation in $440 \quad$ southern Spain and western Anatolia (Turkey). Geophys J Int 121, 337-353.

441 Almendros J, Ibáñez J M, Carmona E, Zandomeneghi D (2007) Array analyses of volcanic earthquakes and tremor recorded at Las Cañadas caldera (Tenerife Island, Spain) during the 2004 seismic activation of Teide volcano. J Volcanol Geotherm Res 160, 285-299.

444 Araña V, Martí J, Aparicio A, García Cacho L, García R (1994) Magma mixing in alkaline 445 magmas: An example from Tenerife, Canary Islands. Lithos 32, 1-19, doi:10.1016/00244937(94)90018-3

Araña V, Camacho A G, García A, Montesinos F G, Blanco I, Vieira R, Felpeto A (2000) 0273(00)00215-8

Blanco-Montenegro I, Nicolosi I, Pignatelli A, García A, Chiappini M(2011) New evidence about the structure and growth of ocean island volcanoes from aeromagnetic data: The case of Tenerife, Canary Islands. $J$ Geophys Res 116 B03102, doi:10.1029/2010JB007646.

O’Connell R J, Budiansky B (1977) Viscoelastic properties of fluid-satured cracked solids. $J$ Geophysical Res 82,: 5719-5735.

457

Canales J P, Dañobeitia J J,Watts A B (2000) Wide-angle seismic constraints on the internal structure of Tenerife, Canary Islands. J Volcanol Geotherm Res 103, 65-81, doi:10.1016/S0377-0273(00)00216-X.

460 Canas J A, Ugalde A, Pujades F G, Carracedo J C, Soler V Blanco M J (1998) Intrinsic and scattering seismic wave attenuation in the Canary Islands. J Geophys Res, 103, B7, 15,037-15,050. 
463

464

465

466

467

468

469

470

471

472

473

474

475

476

477

478

479

480

481

482

483

484

485

486

487

Cagniard, L (1953) Basic theory of the magneto-telluric method of geophysical prospecting. Geophysics 18: 605-635. doi:10.1190/1.1437915.

Carcolé E, Sato H (2010) Spatial distribution of scattering loss and intrinsic absorption of short-period $\mathrm{S}$ waves in the lithosphere of Japan on the basis of the Multiple Lapse Time Window Analysis of Hi-net data. Geophys J Int 180(1), 268-290.

Cerdeña I D, Del Fresno C, Rivera L (2011) New insight on the increasing seismicity during Tenerife's 2004 volcanic reactivation. J Volcanol Geotherm Res 206(1), 15-29.

Coppo N, Schnegg P A, Heise W, Falco P, Costa R (2008) Multiple caldera collapses inferred from the shallow electrical resistivity signature of the Las Cañadas caldera, Tenerife, Canary Islands. J Volcanol Geotherm Res 170, 153-166, doi:10.1016/j.jvolgeores.2007.09.013.

Coppo N, Schnegg P A, Falco P, Costa R (2010) Conductive structures around Las Cañadas caldera, Tenerife (Canary Islands, Spain): a structural control. Geol Acta 8:67-82

De Barros L, Martini F, Bean C J, García-Yeguas A, Ibáñez J M (2012) Imaging magma storage below Teide volcano (Tenerife) using scattered seismic wavefields. Geophys $J$ Int 191:695-706.

De Gori P, Chiarabba C, Patanè D(2005) Qp structure of Mount Etna: constraints for the physics of the plumbing system. J Geophys Res 110(B05303), doi:10.1029/2003JB002875.

De SienaL, Del Pezzo E, Bianco F (2011) A scattering image of Campi Flegrei from the autocorrelation functions of velocity tomograms. Geophys J Int.184, 1304-1310 doi: 10.1111/j.1365-246X.2010.04911.x

De Siena L, Del Pezzo E, Thomas C, Curtis A, Margerin L (2013) Seismic energy envelopes in volcanic media: in need of boundary conditions. Geophys J Int 195, 1102-1119 doi: $10.1093 /$ gji/ggt273 
De Siena L, Thomas C, Waite G P, Moran S C, Klemme S (2014) Attenuation and scattering tomography of the deep plumbing system of Mount St. Helens. J Geophys Res Solid Earth, 119(11), 8223-8238.

De Siena L, Calvet M, Watson K J, Jonkers ART,Thomas C (2016) Seismic scattering and absorption mapping of debris flows, feeding paths, and tectonic units at Mount St. Helens volcano. Earth Planet Sci Letters, 442, 21-31, http://dx.doi.org/10.1016/j.epsl.2016.02.026.

Del Pezzo E, Bianco F, Saccorotti G (2001) Separation of intrinsic and scattering Q for volcanic tremor: an application to Etna and Masaya volcanoes. Geophys Res Letters 28, 3083-3086.

Del Pezzo E, Bianco F, Zaccarelli L (2006) Separation of Qi and Qs from passive data at Mt Vesuvius: a reappraisal of the seismic attenuation estimates. Phys Earth Planet Inter, 159, 202-212.

Del Pezzo E (2008) Chapter 13 Seismic Wave Scattering in Volcanoes. Advances in Geophysics, 50, Pages 353-371, ISSN 0065-2687, ISBN 9780123745095, https://doi.org/10.1016/S0065-2687(08)00013-7

Dóniz J, Romero C, Coello E, Guillén C, Sánchez N, García-Cacho L, García A (2008), Morphological and statistical characterization of recent mafic volcanism on Tenerife (Canary Islands, Spain), J Volcanol Geotherm Res, 173, 185-195, doi:10.1016/j.jvolgeores.2007.12.046.

Dóniz-Páez J (2015) Volcanic geomorphological classification of the cinder cones of


http://dx.doi.org/10.1016/j.geomorph.2014.10.004. 
511 Eberhart-Phillips D, Chadwick M, Bannister S (2008) Three dimensional attenuation 512 structure of central and southern south island, New Zealand, from local earthquakes. $J$ $513 \quad$ Geophys Res 113(B05308), doi:10.1029/2007JB005359.

514 Fehler M (1982) Using dual-well seismic measurements to infer the mechanical properties of $515 \quad$ hot dry rock geothermal system. J Geophys Res 87, 5485-5494.

516 Fehler M, Roberts P, Fairbanks T (1988) A temporal change in coda wave attenuation observed during an eruption of Mount St. Helens. J Geophys Res 93, 4367-4373.

518 Frederiksen A W, Revenaugh J (2004) Lithospheric imaging via teleseismic scattering 519 tomography. Geophys J Int 159, 978-990.

520

521

522

523

524

525

526

527

528

529

530

531

532

533

534

535

García-Yeguas A (2010) Estudio de heterogeneidades laterales de volcanes actives: tomografía sísmica de alta resolución de la Isla de Tenerife, anomalías de propagación de ondas sísmicas de la Isla Decepción y otros efectos. Editorial de la Universidad de Granada,(Spain), ISBN: 978-84-693-5992-1,http://hdl.handle.net/10481/6648.

García-Yeguas A, Koulakov I, Ibáñez J M, RietbrockA(2012) High resolution P wave velocity structure beneath Tenerife Island (Canary Islands, Spain). Geophys J Int 117. doi:10.1029/2011JB008,970

Giampiccolo E, Tuvé T, Patanè D (2006) S-waves attenuation and separation of scattering and intrinsic absorption of seismic energy in southeastern Sicily (Italy). Geophys J Int $165,211-222$

Gottsmann J, Camacho A G, Martí J, Wooller L, Fernández J, García A, Rymer H (2008) Shallow structure beneath the central volcanic complex of Tenerife from new gravity data: Implications for its evolution and recent reactivation. Phys Earth Planet Inter, 168, 212-230, doi:10.1016/j.pepi.2008.06.020.

Gusev A, Lemzikov M (1985) Properties of scattered elastic waves in the lithosphere of Kamchatka: Parameters and temporal variations. Tectonophysics 112, 137-153. 
536 Hartigan J A(1975) Clustering Algorithms, Wiley Series in Probability and Mathematical 537 Statistics. John Wiley \& Sons, New York.

538 Holliger K, Kanamori H, Clayton R W (1996) Upper-crustal seismic velocity heterogeneity $539 \quad$ as derived from a variety of P-wave sonic logs. Geophys J Int, 125, 813-829.

540 Hoshiba M, Rietbrock A, Scherbaum F Nakahara H, Haberland C (2001) Scattering

541

542

543

544

545

546

547

548

549

550

551

552

553

554

555

556

557

558

559

560 attenuation and intrinsic absorption using uniform and depth dependent model Application to full seismogram envelope recorded in Northern Chile. J Seismology 5, 157. doi:10.1023/A:1011478202750

Ibáñez J M, Rietbrock A, García-Yeguas A (2008) Imaging an active volcano edifice at Tenerife Island, Tenerife Island, Spain. Eos Trans AGU, 89(32), 289-290, doi:10.1029/2008EO320001.

Lilliefors H (1967) On the Kolmogorov-Smirnov test for normality with mean and variance unknown. J Am Stat Assoc,62, 399-402.

Mikada H, Watanabe H, Sakashita S (1997) Evidence for subsurface magma bodies beneath Izu-Oshima volcano inferred from a seismic scattering analysis and possible interpretation of the magma plumbing system of the 1986 eruptive activity. Phys Earth Planet Inter 104:257-69

Nishigami K (1997) Spatial distribution of coda scatterers in the crust around two active volcanoes and one active fault system in central Japan: inversion analysis of coda envelope. Phys Earth Planet Inter 104:75-89

Nolet G (2008) A breviary of seismic tomography. Imaging the Interior of the Earth and Sun. Cambridge University Press, 360 pp.

Patanè D, Barberi G, De Gori P, Cocina O, Zuccarello L, Garcia-Yeguas A, Castellano M, D'Alessandro A, Sgroi T (2017) The shallow magma chamber of Stromboli Volcano (Italy). Geophys Res Lett, 44, 6589-6596, doi:10.1002/2017GL073008. 
561 Piña-Varas P, Ledo J, Queralt P, Marcuello A, Bellmunt F, Hidalgo R,Messeiller M (2014) 3D Magnetotelluric Exploration of Tenerife Geothermal System (Canary Islands, Spain). Surv Geophys 35(4),1045-1064.

564

Piña-Varas P, Ledo J, Queralt P, Marcuello A, Bellmunt F, Ogaya X, Pérez N, RodriguezLosadaJ A (2015) Vertical collapse origin of Las Cañadas caldera (Tenerife, Canary Islands) revealed by 3-D magnetotelluric inversion. Geophys Res Lett, 42, doi:10.1002/2015GL063042

568

Prudencio J, Del Pezzo E, García-Yeguas A, Ibáñez J M (2013) Spatial distribution of 569 intrinsic and scattering seismic attenuation in active volcanic islands - I: model and the

Prudencio J, Ibáñez J M, Del Pezzo E, Marti J, Garcia-Yeguas A, De Siena L (2015)a 3D 572 attenuation tomography of the volcanic island of Tenerife (Canary Islands). Surv 573 Geophys, doi: 10.1007/s10712-015-9333-3

574

Prudencio J, De Siena L, Ibáñez J M, Del PezzoE, García-Yeguas A, Díaz-Moreno A (2015)b The 3D attenuation structure of Deception Island (Antarctica). Surv Geophys doi:

Prudencio J, Taira T, Aoki Y, Aoyama H, Onizawa S (2017) Intrinsic and scattering 578 attenuation images of Usu volcano, Japan. Bull Volcanol 79: 29. https://doi.org/10.1007/s00445-017-1117-9

581

Pous J, Heise W, Schnegg P A, Muñoz G, Martí J, Soriano C (2002) Magnetotelluric study of 582 the Las Cañadas caldera Tenerife, Canary Islands: Structural and hydrogeological implications. Earth Planet Sci Lett, 204, 249-263, doi:10.1016/S0012-821X(02)00956-1. 
585 Romero C (1991) Las manifestaciones volcánicas históricas del archipiélago canario. 1463 pp., Cons. de Polít. Territ., Gobierno Autónomo de Canarias, Santa Cruz de Tenerife, Spain.

Romero C (1992) Estudio geomorfológico de los volcanes históricos de Tenerife. 265 pp., Cabildo Insular de Tenerife, Santa Cruz de Tenerife, Spain.

Sánchez-Alzola A, Martí J, García-Yeguas A, Gil A J (2016) Subsidence and current strain patterns on Tenerife Island (Canary Archipelago, Spain) derived from continuous GNSS time series (2008-2015). J Volcan Geotherm Res. http://dx.doi.org/10.1016/j.jvolgeores.2016.08.006

Sato H, Fehler M C, Maeda T (2012) Seismic wave propagation and scattering in the heterogeneous earth (Vol. 496). Berlin: Springer.

Shapiro S A, Hubral P (1999) Elastic Waves in Random Media, Fundamentals of Seismic Stratigraphic Filtering, Springer, Berlin.

Schurr B, Asch G, Rietbrock A, Trumbull R, Haberland C H (2003) Complex patterns of fluid and melt transport in the central Andean subduction zone revealed by attenuation tomography. Earth Planet Sci Lett,215, 105-119.

Schwartz G 1978. Estimating the dimension of a model. Ann. Stat., 6(2), 461-464.

Tramelli A, Pezzo E, Bianco F, Boschi E (2006) 3D scattering image of the Campi Flegrei Caldera (southern Italy). New hints on the position of the old Caldera rim. Phys Earth Planet Int, 155, 269-280.

Valliant H D (1991) Handbook of Geophysical Exploration at Sea. Chapter 7: The Lacoste and Romberg air/sea gravity meter: an overview. Edited by: Geyer R A, Ashwell M. CRC Press. ISBN 0-8493-4252-X 
610 Guzmán J, Iribarren I, Levieux G, López C, Luengo-Oroz N, Martín I, Moreno M, 611 Meletlidis S, Morin J, Moure D, Pereda J, Ricci T, Romero E, Schutze C, Suski-Ricci B, 612 Torres P, Trigo P (2014) Hydrothermal system of central Tenerife volcanic complex, 613 Canary Islands (Spain), inferred from self-potential measurements. $J$ Volcanol Geotherm $614 \quad \operatorname{Res} 272: 59-77$

615 Wegler U, Lühr B G (2001)Scattering behavior at Merapi Volcano (Java) revealed from an 616 active seismic experiment. Geophys J Int 145, 579-592.

617 Wu R S, Xu Z, Li X P(1994) Heterogeneity spectrum and scaleanisotropy in the upper crust 618 revealed by the German Continental Deep Drilling (KTB) Holes. Geophys Res Letters $619 \quad 21(10), 911-914$.

620 Yoon, Mi-Kyung, 2005. Deep seismic imaging in the presence of a heterogeneous 621 overburden. Numerical modelling and case studies from the Central Andes and Southern Andes. Dissertation for PhD. University of Berlin.

623

624

625 